\title{
Rotation curves and dark matter in early type disk galaxies
}

\section{Edo Noordermeer*}

Kapteyn Institute of Astronomy, Groningen, The Netherlands

E-mail: edo@astro.rug.nl

\section{Thijs van der Hulst}

Kapteyn Institute of Astronomy, Groningen, The Netherlands

E-mail: vdhulsteastro.rug.nl

\section{Rob Swaters}

University of Maryland, College Park, USA

E-mail: swaterseastro.umd.edu

We are studying the rotation curves and mass distribution in a sample of 16 early type disk galaxies, with morphological type between S0 and Sab and B-band absolute magnitude between -17 and -22; they form the massive and high-surface brightness extreme of the disk galaxy population. Our study is designed to investigate the relation between dark and luminous matter in these systems, of which little yet is known.

From a combination of WSRT HI observations and long-slit optical spectra, we have obtained high-quality rotation curves which probe the dynamical mass distribution from small to large scales. Due to the presence of the massive bulges, the rotation curves rise extremely rapidly in the centers; even in the optical spectra the central velocity gradients are often barely resolved. Many of our galaxies have declining rotation curves, with the outermost measured velocity up to $40 \%$ lower than the maximum. Some of the galaxies in our sample have very high rotation velocities, with $V_{\max } \sim 400 \mathrm{~km} / \mathrm{s}$.

We decompose the rotation curves into contributions from the luminous (stellar and gaseous) and dark matter. The stellar bulges always dominate the rotation curves in the inner regions and are at least partly responsible for the decline at larger radii. We are able to put both upper and lower limits on their stellar mass-to-light ratios. Dark matter is needed to explain the shape of rotation curves in the outer parts. As an example, we present here the mass models for UGC 9133.

Baryons in Dark Matter Halos

5-9 October 2004

Novigrad, Croatia

${ }^{*}$ Speaker. 


\section{Introduction}

Rotation curves, in particular from HI data extending well outside the optical disks, provide an excellent tool to study the distribution of dark matter in individual disk galaxies. HI observations of early type, massive disk galaxies are however rare. Broeils ([1]) showed a compilation of all highquality HI rotation curves known to that date; only 4 out of 23 galaxies had maximum rotation velocity $V_{\max }>250 \mathrm{~km} / \mathrm{s}$. Since then, many more low- and intermediate mass galaxies have been studied ([过, 月, 丹]), but little progress has been made on the high-mass side. The Universal Rotation Curve of Persic et al. ([\$]) was based on 1100 rotation curves, but only 2 were of type Sab or earlier.

In this study, we address specifically the relation between luminous and dark matter in massive, early type disk galaxies. We investigate whether these systems follow the general trends that exist in later type galaxies (e.g. Tully-Fisher, dark matter content vs. morphological type or surface brightness, etc.), or whether they form a distinct class of galaxies with different characteristics.

\section{Sample selection and observations}

The HI observations are taken from the WHISP survey. The total WHISP survey contains 68 galaxies with morphological type between $\mathrm{S}^{-}$and $\mathrm{Sab}$. From this subsample, we choose by eye the galaxies that are not interacting, have no strong bars, and have well-resolved velocity fields showing ordered rotation. Our final rotation curve sample contains 16 galaxies.

Additional long-slit $\mathrm{H} \alpha$ spectra at high angular resolution were obtained from the INT on La Palma, to circumvent the problem of beam-smearing in the HI data. The stellar mass distribution is determined from multi-color broad-band optical images taken with the JKT on La Palma.

\section{Rotation curve shapes in early type disk galaxies}

In Fig. 1. we show the rotation curves for 9 galaxies from our sample. These rotation curves show some characteristics that are typical for all galaxies in our sample. The rotation curves rise extremely fast in the inner regions. Even in the optical spectra, the rise in rotational velocity is often barely resolved. Total velocity gradients of order $500 \mathrm{~km} / \mathrm{s}$ within $1 \mathrm{kpc}$ are not unusual. After the steep central rise, most rotation curves show a slow but systematic decline. In extreme cases (e.g. UGC 4458) the outer velocity $V_{\text {out }}$ is less than $60 \%$ of the maximum velocity. The rotation velocities in the high-luminosity galaxies are very high: $V_{\max } \sim 400 \mathrm{~km} / \mathrm{s}$. Combined with the large distances out to which we can measure the Hi rotation curves, they imply very high dynamical masses. The total mass inside the outermost point of the rotation curve of UGC 2487 is $2.0 \cdot 10^{12} \mathrm{M}_{\odot}$; to our knowledge, this is the highest mass ever derived from a rotation curve.

The rotation curves presented in Fig. 1 1 look remarkably different from the ones of later-type galaxies, which usually rise slowly and then become flat at large radii. Persic et al. ([5]) claimed that, for a large sample of later type galaxies, the shape of a rotation curve is uniquely determined by a galaxy's luminosity. We have compared our data with their Universal Rotation Curve (URC). The agreement is usually reasonably good in the outer parts, but the URC severely underpredicts the rotation velocities in the central regions. Note that the URC was derived for late type, disk dominated galaxies; the different shape of the rotation curves studied here, compared to those of 


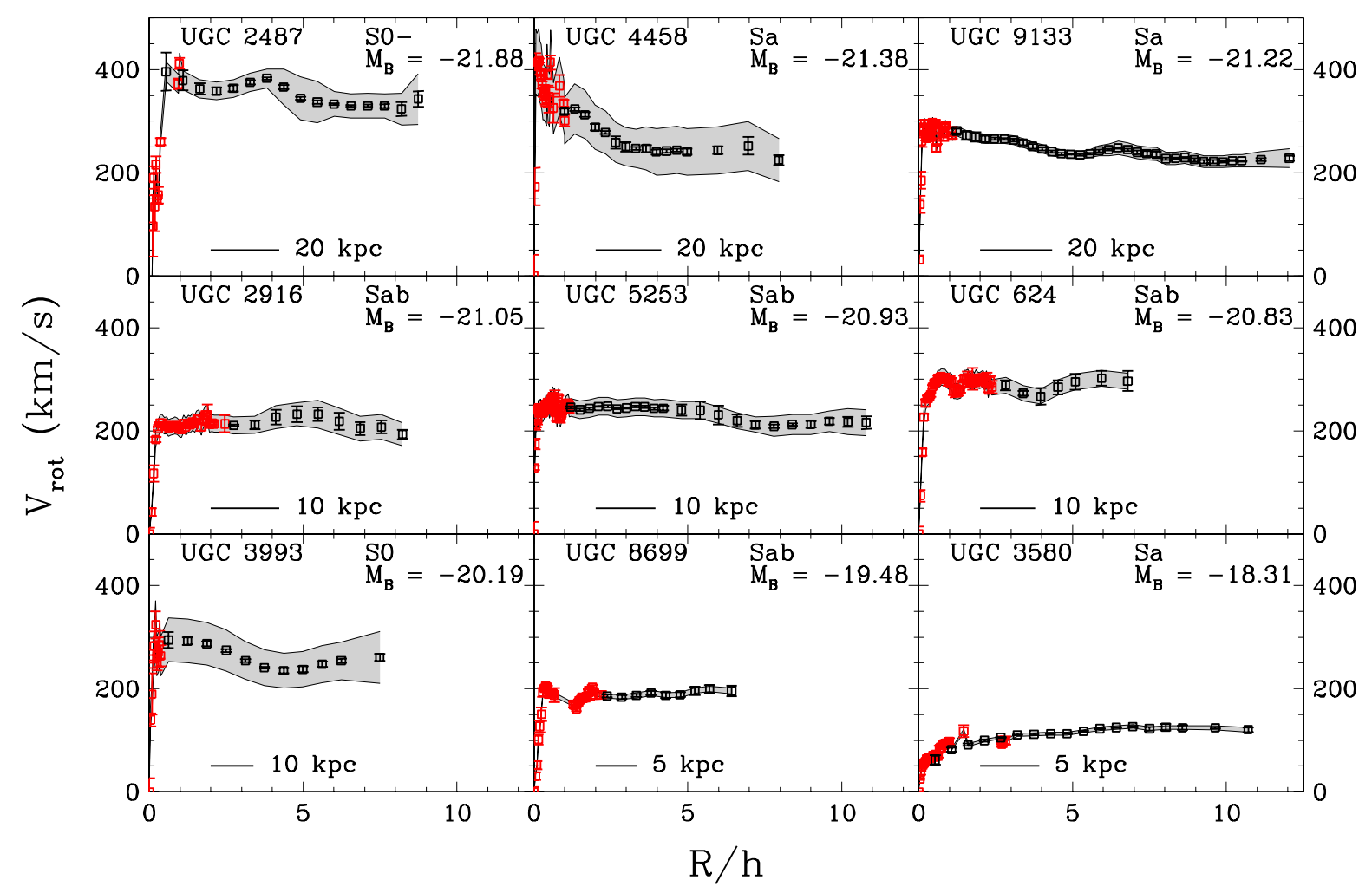

Figure 1: Rotation curves of 9 early type disk galaxies. The morphological type and absolute B-band magnitude are indicated in the top right of each panel. All radii are scaled to the scalelength $h$ of the optical disks, physical scales are indicated with the bars at the bottom of each panel. Black points are derived from tilted-ring fi ts to the HI velocity fi elds, red data points are derived from long slit $\mathrm{H} \alpha$ spectra. The grey shaded bands indicate the uncertainty in rotation velocities due to errors in the determined value for the inclination.

later type spiral galaxies, can be explained as a result of the influence of the massive bulges that are present in these systems. Clearly, the contribution of the bulges needs to be explicitly taken into account when studying the shape of the rotation curves in early type disk galaxies.

\section{Mass modelling: UGC 9133}

The main goal of our study is to get a detailed picture of the mass distribution in individual galaxies. We decompose the rotation curves into contributions from stellar bulges and disks, neutral gas and dark matter. To illustrate the method, we present here the results for UGC 9133. We only show fits with a pseudo-isothermal dark halo; other halo models will be considered at a later stage. As usual there is a degeneracy between the stellar M/L-ratios and the halo parameters. When the $\mathrm{M} / \mathrm{L}$-ratios are large, the dark halo is diffuse, and contributes only at larger radii ('maximum bulge+disk model', upper panels). Note however that, in order to explain the steep rise of the rotation curve in the center and the decline at larger radii, the bulge and disk cannot have very low mass-to-light ratios. In the bottom panels, we show the models with the minimum M/L-ratios that are allowed by the data. Only by invoking exotic, highly concentrated dark matter distributions, can the bulge M/L-ratio be decreased further. Already, the 'minimum bulge+disk model' model shown 


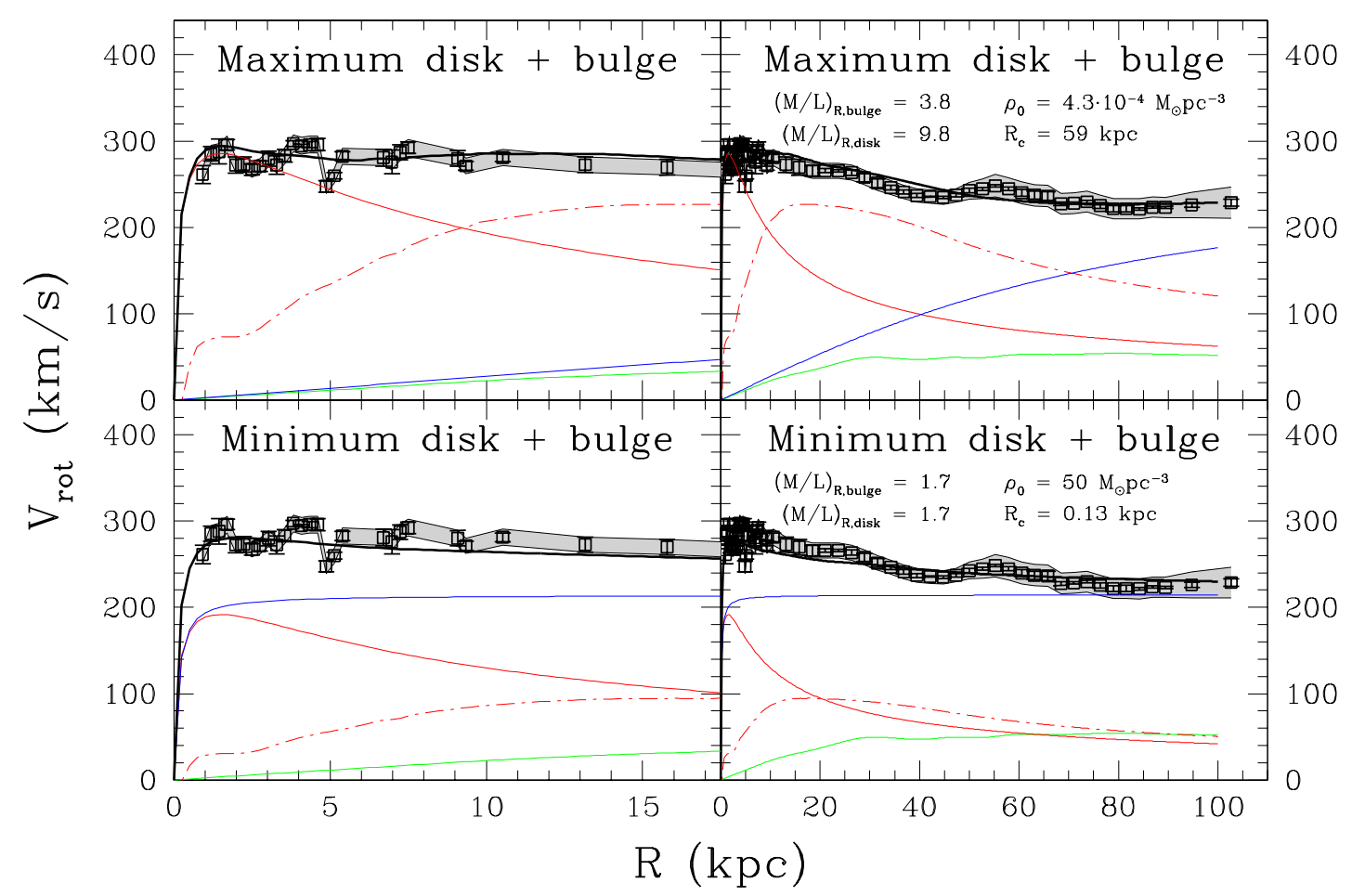

Figure 2: Mass models for UGC 9133. Right hand panels show the full rotation curves, left hand panels are a blow-up of the inner parts. The upper panels show the models with maximal contribution of bulge and disk to the rotation curves, lower panels show the minimal contributions. Symbols with errorbars denote the measured rotation curves, the coloured lines show the contributions of bulge (solid red), disk (dashed red), gas (green) and pseudo-isothermal dark halo (blue). The M/L-ratios for bulge and disk and the halo parameters are given in the right-hand panels. The thick solid line shows the sum of the separate components.

in Fig. 2 has an extremely concentrated dark halo, at odds with current theories of galaxy formation (e.g. [6]). For realistic dark halo concentrations, the M/L-ratio for the bulge must be close to the maximum value shown in the upper panels. For the disk, the constraints on the M/L-ratios are not as tight and realistic dark matter halos can be fitted for a range of disk M/L-ratios. We can, however, still derive lower limits for the disk $\mathrm{M} / \mathrm{L}$ as well; models without a relatively massive disk fail to reproduce the decline of the rotation curve in the outer parts. Additional information on the disk $\mathrm{M} / \mathrm{L}-$ ratio is, however, still required in order to derive the dark matter distribution unambiguously.

\section{References}

[1] Broeils, A.H. 1992, PhD thesis, Rijksuniversiteit Groningen

[2] de Blok, W.J.G., McGaugh, S.S., \& van der Hulst, J.M. 1996, MNRAS, 283, 18

[3] Swaters, R.A. 1999, PhD thesis, Rijksuniversiteit Groningen

[4] Coté, S., Carignan, C., \& Freeman, K.C. 2000, AJ, 120, 3027

[5] Persic, M., Salucci, P., \& Stel, F. 1996, MNRAS, 281, 27

[6] Alam, S.M.K., Bullock, J.S., \& Weinberg, D.H., 2002, ApJ, 572, 34 